\title{
On composite two Higgs doublet models
}

\author{
Enrico Bertuzzo, Tirtha Sankar Ray, Hiroshi de Sandes and Carlos A. Savoy \\ Institut de Physique Théorique, CEA-Saclay, \\ F-91191 Gif-sur-Yvette Cedex, France \\ E-mail: enrico.bertuzzo@cea.fr, tirtha.sankar@unimelb.edu.au, \\ hiroshi.de.sandes@cern.ch, carlos.savoy@cea.fr
}

ABstract: We investigate the issue of anomalous contribution to the $T$ parameter and to Flavor Changing Neutral Currents in models with two Higgs doublets arising as composite pseudo Nambu-Goldstone modes. The non linear Lagrangians of several models are explicitly derived and the anomalous contributions to $\mathrm{T}$ are identified. The breaking patterns $\mathrm{SU}(5) \rightarrow \mathrm{SU}(4) \times \mathrm{U}(1)$ and $\mathrm{SU}(5) \rightarrow \mathrm{SU}(4)$, are analyzed first and we show how anomalous contributions to $\mathrm{T}$ arise in both models. Apart from that, the embedding of the Standard Model fermions in a $\mathbf{1 0}$ of $\mathrm{SU}(5)$ avoids at the same time large corrections to the $Z b \bar{b}$ coupling and Flavor Changing Neutral Current transitions. Finally, we propose a model based on the breaking $\mathrm{SO}(9) / \mathrm{SO}(8)$ that is free from anomalous contributions to $\mathrm{T}$ and in which the problems of the $Z b \bar{b}$ coupling and of Flavor Changing Neutral Currents can be simultaneously solved.

Keywords: Higgs Physics, Beyond Standard Model, Technicolor and Composite Models ArXiv EPRINT: 1206.2623 


\section{Contents}

1 Introduction 1

2 Non linear realization of the Higgs fields 3

$\begin{array}{lll}3 & \text { Gauge interactions } & 6\end{array}$

$\begin{array}{llr}4 & \text { Fermion embeddings } & 8\end{array}$

5 Scalar potential $\quad 11$

6 Other composite 2HDM's: non-linear realizations, PNGB Lagrangian $\begin{array}{lr}\text { and } T \text { parameter } & 12\end{array}$

7 A composite two Higgs doublet model based on $\mathrm{SO}(9) / \mathrm{SO}(8) \quad 15$

8 Conclusions $\quad 16$

A Embedding of the Standard Model fermions in SU(5) representations 17

$\begin{array}{lr}\text { B Algebraic analysis of the } T \text {-parameter } & 18\end{array}$

$\begin{array}{ll}\text { C Embedding of the Standard Model charges in } \mathrm{SO}(9) & 19\end{array}$

D Analysis of the $\mathrm{Sp}(6) / \mathrm{Sp}(4) \times \mathrm{SU}(2)$ coset $\quad 19$

\section{Introduction}

The discovery of a new resonance, with mass of about $125 \mathrm{GeV}$ and properties compatible with those of a Higgs particle [1,2] opens up a new era for particle physics, since the exploration of the Electroweak Symmetry Breaking (EWSB) sector is just at its beginning. A major priority is now the determination of the Higgs properties, i.e. production cross sections and branching ratios. This might shed light on the mechanism of EWSB and remove the veil off any new physics that may control the tera-scale.

If the Higgs has a non trivial substructure, deviations from the Standard Model (SM) couplings may be observed, while underlying symmetries can protect its mass from dangerous quantum corrections. This simple solution to the gauge hierarchy problem is naturally realized if the Higgs fields are pseudo-Goldstone bosons (PNGB) arising from a spontaneous breaking $G \rightarrow H$ due to some strong dynamics at some scale $f$ [3-6]. Once the strong sector is integrated out, the residual effective theory can be described in terms of a nonlinear sigma model with the Higgs belonging to the coset space $G / H$. Interestingly, some 
of these models can be considered as 4-dimensional duals of 5-dimensional gauge-Higgs unified theories realized in $\mathrm{AdS}_{5}$ space. However, the most important phenomenology can be extracted from effective theories with fairly general considerations about the strong sector and in terms of an expansion in the parameter $\epsilon \equiv v / f$, with $v$ the Fermi scale.

Models with minimal [7-9] and non-minimal [10-12] scalar sectors have been studied in the literature. Let us notice that several complications are introduced once non-minimal models are considered. The most important issue is to protect the ratio between the vector boson masses. LEP precision measurements at the $\mathrm{Z}$ pole strongly constraint this ratio, quantified in term of the oblique electroweak parameter $T$. Two Higgs doublet models are notorious for breaking the custodial symmetry that protect the $T$ parameter. The situation is even worse in composite models, which are inherently non-renormalizable implying a less constrained Lagrangian.

In this paper, we address the $T$ parameter and other issues for models with two Higgs doublets (2HDM) arising as composite PNGB. Once the embedding of the SM fermions is defined for each case, the Glashow-Weinberg prescription [13] for natural FCNC of the Higgs couplings has to be checked and we exhibit two models where it is automatically realized. Compositeness is also a source of violations of gauge coupling universality and the models are also selected by their ability to preserve the (relative) agreement of the SM prediction for the $Z \bar{b}_{L} b_{L}$ couplings with experiment. This is ensured by a rule on the embedding of the $b$-quark in the models [15].

The general Lagrangian formalism for the PNGB's was formulated in [17], but in the Minimal Composite Higgs Model (MCHM) based on $\mathrm{SO}(5) / \mathrm{SO}(4)$ [7] the non-linear realization of the symmetry has also been conveniently formulated in terms of a $\mathrm{SO}(5)$ vector reminiscent of a linear realization of the breaking. Because of its utility, we generalize here this approach to the various cosets related to $2 \mathrm{HDM}$ and write in a compact form the PNGB Lagrangian, gauge couplings, Higgs potential and Yukawa couplings in terms of the new variables linearly transforming in fundamental representations. Then, our analysis of the custodial symmetry and tree-level contributions to $T$ for the different cosets defining 2HDM's is simplified by the use of these coordinates. As pointed out in [11] and as also discussed below, in the $\mathrm{Sp}(6) / \mathrm{Sp}(4) \times \mathrm{SU}(2)$ case with a specific embedding of the $\mathrm{SM}$ gauge symmetries, there are no contributions to the $T$ parameter. Here, we also describe another $T$-safe model based on the coset $\mathrm{SO}(9) / \mathrm{SO}(8)$.

For concreteness, we first focus on the symmetry breaking patterns $\mathrm{SU}(5) \rightarrow \mathrm{SU}(4) \times H^{\prime}$ with $H^{\prime}=\mathrm{U}(1)$ or nothing. In the former case, eight pseudo Goldstone modes are delivered, fitting into two SM doublets. In the latter scenario, the breaking leads to an additional SM singlet. We find how the Higgs couplings to $W$ bosons and fermions are modified with respect to those of the SM Higgs boson, so that different production and decay rates are expected. However, in both cases, only by aligning the vacuum expectation values (vev) of the two Higgs the contribution to $T$ could vanish. The lowest dimensional representations of $\mathrm{SU}(5), \mathbf{5}$ and $\mathbf{1 0}$, provide a natural embedding for the SM fermions. We perform a systematic study of all the possibilities, clearly differentiating between cases that can be successfully embedded into a $5 \mathrm{~d}$ completion and those in which this is not so straightforward. We find that the embedding of the SM fermions in the $\mathbf{1 0}$ can lead to 
a simultaneous resolution of the usual problems of anomalous $Z \bar{b}_{L} b_{L}$ [15] and the flavor problems [16]. The contribution to the (Coleman-Weinberg) PNGB potential from these fermions is displayed and, in general, does not have the symmetry to align the Higgs vev's.

Finally, we propose a $\mathrm{SO}(9) / \mathrm{SO}(8)$ model, where the two Higgs couplings to the gauge bosons are much like in the MCHM and, indeed, there are no tree-level contribution to the $T$ parameter. Another interesting feature of this model is an embedding of the SM symmetries in $\mathrm{SO}(8)$, which preserve an extra $\mathrm{U}(1)$ that requires the $\mathrm{SM}$ fermions to couple to only one Higgs doublet. This avoids FCNC problems from the Higgs sector since the other PNGB doublet becomes inert. The $Z \bar{b}_{L} b_{L}$ issue is also coped with when all the fermions are embedded in the spinorial representations. We also survey the different C2HDM that have been studied in the literature in our framework and compare and contrast them with the new models presented here.

The paper is organized as follows. In sections $2-3$ we state the problem and set the notation studying the coset and discussing the gauge interactions for $\mathrm{SU}(5) / \mathrm{SU}(4) \times \mathrm{U}(1)$ and $\mathrm{SU}(5) / \mathrm{SU}(4)$. In section 4 we discuss in detail the possible embeddings for the SM fermions in $\mathrm{SU}(5)$ representations and their phenomenological consequences. In section 5 we study the scalar potential in the $\mathrm{SU}(5)$ case. In section 6 we introduce the gauge interactions and the tree level contribution to the $T$ parameter for different cosets. In section 7 we analyze the $\mathrm{SO}(9) / \mathrm{SO}(8)$ gauge and fermion couplings to the Higgs sector. Finally we conclude with some general observations.

\section{Non linear realization of the Higgs fields}

The general formulation of non linear representations (n.l.r.) is given in CCWZ. Here we introduce an alternative formulation which has also been used in models with a single composite Higgs doublet like those quoted above.

We begin with the study the breaking patterns $\mathrm{SU}(5) / \mathrm{SU}(4)$ and $\mathrm{SU}(5) / \mathrm{SU}(4) \times \mathrm{U}(1)$. The coset space of the two cases is quite similar, although they contain a different number of Goldstone modes. The Goldstone modes can be parametrized as the matrix

$$
\Pi=\left(\begin{array}{c|c}
\frac{1}{\sqrt{20}} \phi_{0} \mathbf{1}_{4} & \Phi \\
\hline \Phi^{\dagger} \tilde{\Phi}^{\dagger} & -\frac{4}{\sqrt{20}} \phi_{0}
\end{array}\right),
$$

where $\Phi$ and $\tilde{\Phi}$ are written in terms of two complex $\mathrm{SU}(2)_{L}$ doublets $\phi_{1}, \phi_{2}$ as

$$
\Phi \equiv \frac{\phi_{1}+i \phi_{2}}{\sqrt{2}} \text { and } \tilde{\Phi} \equiv \frac{\tilde{\phi}_{1}+i \tilde{\phi}_{2}}{\sqrt{2}}
$$

with $\tilde{\phi}_{i}=i \sigma_{2} \phi_{i}^{*}$ (see appendix A for the conventions on the generators of the group). Each $\left(\phi_{i}, \tilde{\phi}_{i}\right)$ transforms as a $(\mathbf{2}, \mathbf{2})$ of $\mathrm{SU}(2)_{L} \times \mathrm{SU}(2)_{R}$, while the $9^{\text {th }}$ Goldstone boson, the singlet $\phi_{0}$, is present only in the $\mathrm{SU}(5) / \mathrm{SU}(4)$ coset. Correspondingly, the Goldstone boson matrix for the symmetric coset $\mathrm{SU}(5) / \mathrm{SU}(4) \times \mathrm{U}(1)$ is obtained for $\phi_{0}=0$. The matrix $\Pi$ parametrizes the elements of the cosets in terms of the PNGB fields. 
To write the n.l.r. of the Higgs fields, it is convenient to choose a specific direction for the vacuum $\Sigma_{0}$. The breaking $\mathrm{SU}(5) \rightarrow \mathrm{SU}(4)$ can be parametrized either through the fundamental or the symmetric representation of $\mathrm{SU}(5)$,

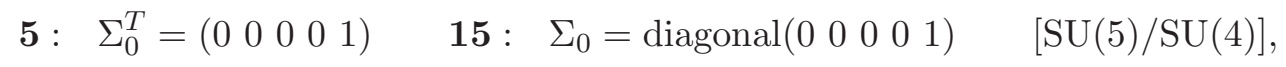

while $\mathrm{SU}(5) \rightarrow \mathrm{SU}(4) \times \mathrm{U}(1)$ can be parametrized through the adjoint representation,

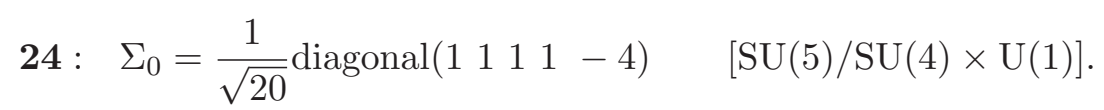

The expressions for the n.l.r. in terms of the Goldstone bosons in the two cases are given by

$$
\begin{aligned}
\text { 5: } & \Sigma=e^{\frac{i \Pi}{f}} \Sigma_{0} \\
15: & \Sigma=e^{\frac{i \Pi}{f}} \Sigma_{0} e^{\frac{i \Pi T}{f}} \\
24: & \Sigma=e^{\frac{i \Pi}{f}} \Sigma_{0} e^{\frac{-i \Pi}{f}}
\end{aligned}
$$

where the appropriate vacuum $\Sigma_{0}$ must be used and $f$ is the scale at which the breaking occurs.

The n.l.r. is obtained in terms of a 5-components unit vector $u$ as follows. In the $\mathrm{SU}(5) / \mathrm{SU}(4)$ case, $u$ reads

$$
u=e^{-i \frac{3}{5} \frac{\phi_{0}}{f}}\left(\begin{array}{c}
i \frac{\sin \left(\frac{\varphi}{f}\right)}{\varphi}\left(\begin{array}{c}
\Phi \\
\tilde{\Phi}
\end{array}\right) \\
\cos \left(\frac{\varphi}{f}\right)+i \phi_{0} \frac{\sin \left(\frac{\varphi}{f}\right)}{\varphi}
\end{array}\right)=e^{\frac{i \Pi \Pi}{f}}\left(\begin{array}{l}
0 \\
0 \\
0 \\
0 \\
1
\end{array}\right)=e^{\frac{i \Pi}{f}} u_{0}, \quad u^{\dagger} u=1,
$$

where $\varphi=\sqrt{|\Phi|^{2}+|\tilde{\Phi}|^{2}+\phi_{0}^{2}}$ and

$$
\Sigma=u[\mathbf{5}], \quad \Sigma=u u^{T} \quad[\mathbf{1 5}]
$$

In the $\mathrm{SU}(5) / \mathrm{SU}(4) \times \mathrm{U}(1)$ case, with $\phi_{0}=0$,

$$
u=\left(\begin{array}{c}
i \frac{\sin \left(\frac{\varphi}{f}\right)}{\varphi}\left(\begin{array}{c}
\Phi \\
\tilde{\Phi}
\end{array}\right) \\
\cos \left(\frac{\varphi}{f}\right)
\end{array}\right)=e^{\frac{i \Pi}{f}} u_{0}, \quad u^{\dagger} u=1,
$$

where now $\varphi=\sqrt{|\Phi|^{2}+|\tilde{\Phi}|^{2}}$. In this case the $\Sigma$ matrix reads

$$
\Sigma=-\frac{\sqrt{5}}{2}\left(u u^{\dagger}-\frac{\mathbf{1}_{5}}{5}\right)
$$

Because $\varphi$ is invariant under the preserved $\mathrm{SU}(4), u$ linearly transforms as a $\mathbf{4}+\mathbf{1}$, like the Goldstone fields in eq. (2.1). For the $\mathrm{SU}(5) / \mathrm{SU}(4) \times \mathrm{U}(1)$ case, the $\mathrm{U}(1)$ invariance is obtained by the multiplication of a phase defined by the transformation of the Goldstone 
bosons: $u \rightarrow g u e^{i \theta}$, with $\theta=\theta\left(g, \phi^{i}\right)$. This corresponds to the transformation of $e^{\frac{i \Pi}{f}}$ defined in CCWZ once the SU(4) invariance of vacuum is taken into account. This phase disappears in the realization $\Sigma$ as defined in eq. (2.7).

It is useful to formulate the Lagrangian for the PNGB fields and their interactions ${ }^{1}$ in terms of $u$. First note that $\Sigma \Sigma^{\dagger}$ are projectors along the vector $u$ for the $\mathbf{5}$ and the $\mathbf{1 5}$ vacua. It implies that the insertions of the $\Sigma$ field in the different terms of the effective Lagrangian are quite limited. In practice one has only to introduce the minimal number of $u$ 's needed to ensure the $\mathrm{SU}(5)$ invariance. This is equivalent to the insertion of the unitary matrix $U=e^{\frac{i \Pi}{f}}$ in the CCWZ formalism. However, the breaking by a 15 preserves an additional parity symmetry: $u \rightarrow-u$. Therefore the u's must come by pairs, hence as $\Sigma=u u^{T}$. For the $\mathbf{2 4}$ case, let us remark that the unit matrix in eq. (2.7) is irrelevant as it does not introduce any new operator in the effective Lagrangian. Therefore we henceforth replace eq. (2.7) by $\Sigma=u u^{\dagger}$, which is a projector, $\Sigma^{2}=\Sigma$. Therefore, the effective Lagrangian should only have the minimal number of insertions of the $\Sigma$ field as needed for the $\mathrm{SU}(5)$ invariance in each interaction, much like in the previous case. This drastically simplifies the analysis of flavour changing effects from Higgs couplings to fermions below.

We now turn to the analysis of the Goldstone boson Lagrangians. This can be done in terms of the $u$ 's transforming in the fundamental representation of $\mathrm{SU}(5)$, the simplest n.l.r., from which the other ones can be built. Of course, the Lagrangians for the Goldstone bosons depend only on the specific coset as shown in CCWZ. We easily determine the explicit Lagrangians in terms of the $u$ 's by just imposing the equivalence with the general CCWZ expression. There are two SU(5) invariants with two derivatives since $u^{\dagger} u=1$ :

$$
\begin{aligned}
\partial_{\mu} u^{\dagger} \partial^{\mu} u & =u_{0}^{\dagger} \partial_{\mu} U^{\dagger}\left(U U^{\dagger}\right) \partial^{\mu} U u_{0} \\
u^{\dagger} \partial_{\mu} u \partial^{\mu} u^{\dagger} u & =u_{0}^{\dagger} U^{\dagger} \partial_{\mu} U u_{0} u_{0}^{\dagger} \partial^{\mu} U^{\dagger} U u_{0}
\end{aligned}
$$

where $U=e^{\frac{i \Pi}{f}}$. Notice the projections along the $u_{0}$ direction. The element of the $\operatorname{SU}(5)$ algebra $U^{\dagger} \partial_{\mu} U$ can be expanded as, $i d_{\mu}^{i} T^{i}+i E_{\mu}^{a} T^{a}+(d / E)_{\mu}^{0} T^{0}$, where $T^{a}$ are the generators of $\mathrm{SU}(4), T^{0}$ the one of the $\mathrm{U}(1)$ and $T^{i}$ those of the coset. The component along the $\mathrm{U}(1)$ generator is $d_{\mu}^{0}$ for $\mathrm{SU}(5) / \mathrm{SU}(4)$, and $E_{\mu}^{0}$ for $\mathrm{SU}(4) \times \mathrm{U}(1)$. The $d_{\mu}$ 's transform linearly under local SU(4) and $\mathrm{SU}(4) \times \mathrm{U}(1)$, respectively, while the $E_{\mu}$ 's transform as gauge fields $[17,18]$.

Replacing these expression in eq. (2.8) and projecting along $u_{0}$, one gets for the two invariants two different expressions in terms of $d_{\mu}^{i} d^{\mu i}$ and $E_{\mu}^{0} E^{\mu 0}$. However, since $u_{0}$ is a $\mathrm{SU}(4)$ singlet, the correspondent $E_{\mu}^{a} E^{\mu a}$ terms are absent in both the global invariants in eq. (2.8), while the terms $d_{\mu}^{i}$ transforming as $4+\overline{4}$ are absent from the quartic one. Then, the CCWZ Lagrangian, $\mathcal{L}_{\mathrm{PNGB}}=\Sigma_{i} d_{\mu}^{i} d^{\mu i}$ is given in each case by the combination that eliminates or consistently normalizes the singlet term, resulting in local SU(4) invariant two-derivative PNGB Lagrangians. They are as follows:

$$
\begin{array}{rll}
\mathcal{L}_{\mathrm{PNGB}} & =f^{2}\left(\partial^{\mu} u^{\dagger} \partial_{\mu} u-u^{\dagger} \partial_{\mu} u \partial^{\mu} u^{\dagger} u\right) & {[\mathrm{SU}(5) / \mathrm{SU}(4) \times \mathrm{U}(1)]} \\
& =f^{2}\left(\partial^{\mu} u^{\dagger} \partial_{\mu} u-\frac{3}{8} u^{\dagger} \partial_{\mu} u \partial^{\mu} u^{\dagger} u\right)[\mathrm{SU}(5) / \mathrm{SU}(4)]
\end{array}
$$

\footnotetext{
${ }^{1}$ For a review of the more usual CCWZ approach in the context of EWSB see, e.g., [18].
} 


\begin{tabular}{|c|c|c|}
\hline Vertex & 2 HDM & C2HDM \\
\hline$h W^{+} W^{-} / Z Z$ & $2 \frac{m_{W, Z}^{2}}{v}$ & $2 \frac{m_{W, Z}^{2}}{v} \sqrt{1-\epsilon^{2}}$ \\
\hline$h^{2} W W / Z Z$ & $\frac{m_{W, Z}^{2}}{v^{2}}$ & $\frac{m_{W, Z}^{2}}{v^{2}}\left(1-2 \epsilon^{2}\right)$ \\
\hline$H^{2} W W / Z Z$ & $\frac{m_{W, Z}^{2}}{v^{2}}$ & $\frac{m_{W, Z}^{2}}{v^{2}} r_{\epsilon} \sqrt{1-\epsilon^{2}}$ \\
\hline$A^{2} W W$ & $\frac{m_{W}^{2}}{v^{2}}$ & $\frac{m_{W}^{2}}{v^{2}} r_{\epsilon} \sqrt{1-\epsilon^{2}}$ \\
\hline$A^{2} Z Z$ & $\frac{m_{Z}^{2}}{2 v^{2}}$ & $\frac{1}{2} \frac{m_{Z}^{2}}{v^{2}}\left(r_{\epsilon} \sqrt{1-\epsilon^{2}}-2 \epsilon^{2} r_{\epsilon}^{2}\right)$ \\
\hline$H^{+} H^{-} W^{+} W^{-}$ & $2 \frac{m_{W}^{2}}{v^{2}}$ & $2 \frac{m_{W}^{2}}{v^{2}}\left(r_{\epsilon} \sqrt{1-\epsilon^{2}}-2 \epsilon^{2} r_{\epsilon}^{2}\right)$ \\
\hline$H^{+} H^{-} Z Z$ & $2 \frac{m_{Z}^{2}}{v^{2}}\left(1-2 \frac{m_{W}^{2}}{m_{Z}^{2}}\right)^{2}$ & $2\left[\frac{m_{Z}^{2}}{v^{2}} r_{\epsilon} \sqrt{1-\epsilon^{2}}-\right.$ \\
\hline$H^{-} W^{+} A_{\mu}(H+i A)$ & $-\frac{g e}{2}$ & $\left.-4 \frac{m_{W}^{2}}{v^{2}}\left(1-\frac{m_{W}^{2}}{m_{Z}^{2}}\right) r_{\epsilon}^{2}\right]$ \\
\hline$H^{-} W^{+} Z H$ & $\frac{g^{\prime} e}{2}$ & $-\frac{g e}{2} r_{\epsilon}^{2}$ \\
\hline$H^{-} W^{+} Z A$ & $i \frac{g^{\prime} e}{2}$ & $\frac{g^{\prime} e}{2} r_{\epsilon}^{2}$ \\
\hline$H^{+} H^{+} W^{-} W^{-}$ & $\times$ & $\kappa \frac{g^{\prime} e}{2} r_{\epsilon}^{2}\left[1-2 \kappa \epsilon^{2}\left(1+\frac{g^{2}}{g^{\prime 2}}\right)\right]$ \\
\hline
\end{tabular}

Table 1. Gauge interaction for the Higgs fields in the C2HDM case following from eq. (3.1), as opposed to the usual renormalizable $2 \mathrm{HDM}$ case. The Higgs fields definition is given in eq. (3.2). We used the shorthanded notation $\epsilon=v / f$ and $r_{\epsilon}=\epsilon / \arcsin (\epsilon)$.

Notice that in the first lagrangian in (2.9) there is only one invariant under local $\mathrm{SU}(4)$, while the second Lagrangian differs by the presence of a second one, $d^{0} d^{0}=$ $(5 / 8) u^{\dagger} \partial_{\mu} u \partial^{\mu} u^{\dagger} u$, since with our normalization of $T^{0}, T^{0} u_{0}=-\sqrt{8 / 5}$.

It is worth remarking that the use of the $u$ variables and the projection into the $u_{0}$ automatically eliminates most of the $E_{\mu}$ 's, towards the construction of the locally invariant Lagrangians. This procedure is generalized in section 6 to other cosets. If useful, the corresponding expressions for the $\Sigma$ 's can be obtained by using their expressions in terms of the $u$ 's. In the next section we show that the anomalous contribution to the T-term is generally encoded in the quartic term in the $u$ 's of the Lagrangian.

\section{Gauge interactions}

The gauge Lagrangian for the Goldstone bosons in the effective theory can be written from eq. (2.9) introducing covariant derivatives for the $u$ variables, $\partial u_{i} \rightarrow \partial u_{i}-i(\hat{W} u)_{i}$, with $\hat{W}_{\mu}=g W_{\mu}^{+} T_{L}^{-}+g W_{\mu}^{+} T_{L}^{-}+e A_{\mu}\left(T_{L}^{3}+T_{R}^{3}\right)+g \sec \theta_{W} Z_{\mu}\left(T_{L}^{3}-\tan ^{2} \theta_{W} T_{R}^{3}\right)$. Therefore the coupling of the SM gauge bosons to the PNGB fields contains two algebraically different terms,

$$
u^{\dagger} \hat{W}_{\mu} \hat{W}_{\nu} u-\kappa u^{\dagger} \hat{W}_{\mu} u u^{\dagger} \hat{W}_{\nu} u
$$


and only the first one, quadratic in $u$, is analogous to the SM.

The gauge interactions following from the previous expression are given in table (1), with Higgs fields defined as

$$
\phi_{1}=\left(\begin{array}{c}
G^{+} \\
h+i G^{0}
\end{array}\right) \quad \phi_{2}=\left(\begin{array}{c}
H^{+} \\
H+i A
\end{array}\right) .
$$

and $G^{0,+}$ the electroweak Goldstone bosons. It is interesting to note that different couplings between the Higgs boson and the $\mathrm{W}$ and $\mathrm{Z}$ vectors could be important to explain the crosssection and branching ratios of the observed excess around $125 \mathrm{GeV}[20]$.

In momentum space, the gauge Lagrangian reads

$$
\mathcal{L}_{\text {gauge }}=\frac{1}{2} p_{\mu \nu}^{T}\left[\Pi_{0}\left(q^{2}\right) \operatorname{tr}\left(\hat{W}_{\mu} \hat{W}_{\nu}\right)+\Pi_{1}\left(q^{2}\right)\left(u^{\dagger} \hat{W}_{\mu} \hat{W}_{\nu} u-\kappa u^{\dagger} \hat{W}_{\mu} u u^{\dagger} \hat{W}_{\nu} u\right)\right]
$$

where $\kappa=1$ or $3 / 8$ as in eq. (2.9) and $\Pi_{0}\left(q^{2}\right)$ and $\Pi_{1}\left(q^{2}\right)$ are form factors. Of course, this Lagrangian is not invariant under $\mathrm{SU}(2)_{L} \times \mathrm{SU}(2)_{R} \subset \mathrm{SU}(4)$ because only the SM subgroup is gauged. In $2 \mathrm{HDM}$ 's, only the alignment between the two doublets in the vacuum ensures a custodial $\mathrm{SU}(2)$ to protect the $T$ parameter.

Indeed, in both the cases under consideration the quartic term spoils the custodial symmetry already at tree level. To investigate this crucial point, let us keep in eq. (3.3) only the components of $u$ with electric charge $T_{L}^{3}+T_{R}^{3}=0$, namely, $u_{2}$ and $u_{3}$ with the conventions defined in appendix A. The embedding of the $\mathrm{SU}(2)_{L} \times \mathrm{U}(1)_{Y}$ generators in $\mathrm{SU}(2)_{L} \times \mathrm{SU}(2)_{R} \subset \mathrm{SU}(4)$ is uniquely defined by $\boldsymbol{4}=\mathbf{( 2 , 2 )}$, so that the masses of the gauge bosons are obtained from eq. (3.3) as follows:

$$
\mathcal{L}_{0}=f^{2}\left(\left\langle\left|u_{2}\right|^{2}\right\rangle+\left\langle\left|u_{3}\right|^{2}\right\rangle\right) \frac{g^{2}}{2}\left(W_{\mu}^{+} W_{\mu}^{-}+\frac{1}{2 \cos ^{2} \theta_{W}}\left(1-\kappa \frac{\left(\left\langle\left|u_{2}\right|^{2}\right\rangle-\left\langle\left|u_{3}\right|^{2}\right\rangle\right)^{2}}{\left\langle\left|u_{2}\right|^{2}\right\rangle+\left\langle\left|u_{3}\right|^{2}\right\rangle}\right) Z_{\mu} Z_{\mu}\right) .
$$

The additional contribution to the $\mathrm{Z}$ mass only vanishes for $\left\langle\left|u_{2}\right|\right\rangle=\left\langle\left|u_{3}\right|\right\rangle$, which is precisely the condition for the alignment of the two doublets in the vacuum and the conservation of the custodial symmetry. Since the PNGB potential is generated at loop level, where $\mathrm{SU}(2)_{L} \times \mathrm{SU}(2)_{R} \subset \mathrm{SU}(4)$ is explicitly broken, it cannot generically guarantee such a degeneracy for the solution, so that a large contribution to the $T$ parameter should be present already at tree level. This would impose an unnatural hierarchy between the two relevant scales, $f \gg v$ (slightly less for $\kappa=3 / 8$ ).

In principle: $i$ ) the custodial symmetry could be effective in a region of the parameter space for the solution of the equations of motion, and $i i$ ) the contributions to the $T$ parameter (both at tree and loop level) from the scalar sector can be partially compensated by fermionic contributions [19]. Nevertheless, the mechanism for this approximate cancellation must be natural.

The conditions to avoid Higgs misalignments in composite 2HDM have been exhaustively discussed in [11], in particular, by the use of additional discrete symmetries. They were able to build a model with one Higgs coupled to the SM fermions and an inert Higgs that does not contribute to the gauge boson masses. The natural discrete symmetry for 
$\mathrm{SU}(5)$ is charge conjugation ( $\phi_{1} \rightarrow \phi_{1}$ and $\phi_{2} \rightarrow-\phi_{2}$, see eq. (2.2)). To investigate this point, as much as the mostly important flavour issues, we now turn to a study of the SM fermion embedding and their couplings to the two Higgs bosons.

\section{Fermion embeddings}

As usual in Composite Higgs models, the coset structure does not fix the embedding of the fermions in $G$ representations. Moreover, different choices will in principle generate different terms in the Coleman-Weinberg potential. However, some general considerations allow to make the model theoretically consistent and phenomenologically viable. Some of the important issues are:

1. LEP has measured the $Z b_{L} \bar{b}_{L}$ coupling with high precision, finding results in agreement with the SM prediction at nearly $0.25 \%$ level. $^{2}$ As shown in [15], a custodial symmetry can protect this coupling from large contributions due to composite states. The argument boils down to the conclusion that the left handed quark doublet (at least the third generation) should be embedded in a $(\mathbf{2}, \mathbf{2})_{\mathbf{2} / \mathbf{3}}$ representation of $\mathrm{SU}(2)_{L} \times \mathrm{SU}(2)_{R} \times \mathrm{U}(1)_{X}$ in order to protect this coupling from getting too large corrections.

2. If the $\mathrm{SM}$ fermions can be embedded into $G$ representations in a unique way, then the problem of FCNC is considerably reduced $[10,16]$. There are cases where this can be achieved by the addition of discrete symmetries [11]. In the $\mathrm{SO}(9) / \mathrm{SO}(8)$ model described below this is done by a $\mathrm{U}(1) \in \mathrm{SO}(8)$.

3. In $5 \mathrm{~d}$ models of gauge-Higgs unification that are perturbative dual to the strongly coupled $4 \mathrm{~d}$ model, where the PNGB are the 5-th component of gauge bosons, the fermions of both chiralities should be in the same representation of the gauge group (global group, from the $4 \mathrm{~d}$ point of view). ${ }^{3}$ However, this is not a stringent consideration for the purely $4 \mathrm{~d}$ model, and we will actually explore some of the advantages of using $\mathrm{LH}$ and $\mathrm{RH}$ fermions in different representations.

In terms of the SM fermions, the Lagrangian in momentum space reads [7]

$$
\begin{aligned}
\mathcal{L}_{\text {fermion }}= & \bar{q}_{L} \not p\left[\Pi_{0}^{q}+\Pi_{1}^{q} g_{q_{L}}\left(u_{i}\right)\right] q_{L}+ \\
& +\bar{t}_{R} \not p\left[\Pi_{0}^{u}+\Pi_{1}^{u} g_{t_{R}}\left(u_{i}\right)\right] t_{R}+\bar{b}_{R} \not p\left[\Pi_{0}^{d}+\Pi_{1}^{d} g_{b_{R}}\left(u_{i}\right)\right] b_{R}+ \\
& +f \bar{t}_{R}\left[M_{1}^{u} g_{t q}\left(u_{i}\right)\right] q_{L}+f \bar{b}_{R}\left[M_{1}^{d} g_{b q}\left(u_{i}\right)\right] q_{L}
\end{aligned}
$$

where the $\Pi_{i}\left(Q^{2}\right)$ and $M_{i} Q^{2}$ are form factors controlled by the strong sector and the $g\left(u_{i}\right)$ functions of the Higgs fields. These polynomials are defined by the possible insertions of

\footnotetext{
${ }^{2}$ Recent 2 loop calculations indicates that the agreement between SM and experimental values of the $Z b_{L} \bar{b}_{L}$ coupling may not be as good as previously expected [14].

${ }^{3}$ In principle, this requirement can be circumvented by introducing extra fermion multiplets and mass terms in the IR brane. However, this is a potential source of FCNC, so that we discard this assemblage in our discussion.
} 
the n.l.r. $u$ 's, or $\Sigma$ 's in the fermion bilinear terms $\bar{\Psi}_{A}^{i} g^{i j}(u) \Psi_{B}^{j}$, with $A, B=L, R$ that are $\mathrm{SU}(5)$ invariants. The Lagrangian is obtained by restricting the fermion multiplets to their SM components.

For simplicity, in what follows we will limit ourselves to the lower dimensional representations of SU(5), i.e. $\mathbf{5}$ and $\mathbf{1 0}$, although higher dimensional representations in principle can be used. In terms of $\mathrm{SU}(2)_{L} \times \mathrm{SU}(2)_{R}$ they read

$$
\begin{aligned}
\mathbf{5} & =(\mathbf{2}, \mathbf{2})+(\mathbf{1}, \mathbf{1}) \\
\mathbf{1 0} & =(\mathbf{2}, \mathbf{2})+(\mathbf{3}, \mathbf{1})+(\mathbf{1}, \mathbf{3})
\end{aligned}
$$

Let us first discuss the case in which both the left $(\mathrm{LH})$ and right $(\mathrm{RH})$ fermions are embedded in the fundamental representation. To accommodate hypercharge, each $\mathrm{RH}$ singlet must be embedded in an independent 5, with appropriate X-charge. This implies that, by $\mathrm{U}(1)_{X}$ invariance, $q_{L}$ must be associated to two $\mathbf{5}$, respectively $\Psi_{L}^{t}$ and $\Psi_{L}^{b}$. In a $5 \mathrm{D}$ picture, this is achieved decoupling the additional degrees of freedom through a mass term in the brane $[8,10]$. However, since $b_{L}$ does not belong to $(\mathbf{2}, \mathbf{2})_{\mathbf{2} / \mathbf{3}}$, the $Z b_{L} b_{L}$ vertex is not protected. In models with only one Higgs doublet, this is not necessarily a shortcoming since the breaking is proportional to the relatively small bottom Yukawa coupling [10]. In $2 \mathrm{HDM}$ this is a cause for concern for large $\tan \beta$.

Turning to the case in which both the LH and RH fermions are embedded in a 10, the RH singlets must be in a unique $(\mathbf{1}, \mathbf{3}) \in \mathbf{1 0}_{2 / 3}$, with $T_{3 R}=0$ for $u_{R}$ and $T_{3 R}=-1$ for $d_{R}$. As a consequence, also $q_{L}$ is embedded in a unique representation and $Z b_{L} b_{L}$ is protected. This is the SU(5) counterpart of the choice of the real $\mathbf{1 0}$ for the fermions in the $\mathrm{SO}(5) / \mathrm{SO}(4)$ model.

In both cases, there is only one possible embedding of the SM fermions in the $\mathrm{SU}(2)_{L} \times$ $\mathrm{SU}(2)_{R}$ representations. Another important feature: for all $\bar{\Psi}_{A}^{i} g^{i j}(u) \Psi_{B}^{j}$, we find $g^{i j}(u) \propto$ $u u^{\dagger}=\Sigma$. Indeed, $\Sigma$ is a projector, $\Sigma^{2}=\Sigma$, and $\Sigma \Psi_{L, R}^{i} \Sigma=0$ for the SM in a 10. The expressions for the $g$ functions, as defined in eq. (4.1), are collected in table 2 for both $\mathrm{SU}(5) / \mathrm{SU}(4)$ and $\mathrm{SU}(5) / \mathrm{SU}(4) \times \mathrm{U}(1)$, choosing the appropriate $u$ in eqs. (2.4)(2.6). A crucial attribute of the mass terms is that the up quarks get masses only from one combination of the two Higgs, while the down quarks couple to the orthogonal one. Therefore there is no flavour violation from the Higgs couplings.

In all the previous cases, a 5-dimensional gauge-Higgs unification can be consistently constructed. However, from a purely $4 \mathrm{~d}$ perspective, the embedding of $\mathrm{LH}$ and $\mathrm{RH}$ fermions in different representations make sense, so that we will now briefly comment also on this possibility. Since we are confining ourselves only to the $\mathbf{5}$ and $\mathbf{1 0}$, only for SU(5)/SU(4) the embedding in different representations is possible. The simplest possibilities are given by:

(a) $\left(\Psi_{L}, \Psi_{R}\right) \in(\mathbf{5}, \mathbf{1 0})$, with invariant $\mathbf{5}_{\Sigma}^{T} \overline{\mathbf{1 0}}_{R} \mathbf{5}_{L}$. Note that left doublet can be embedded in the $(\mathbf{2}, \mathbf{2})_{\mathbf{2} / \mathbf{3}}$, protecting $Z b_{L} \bar{b}_{L}$.

(b) $\left(\Psi_{L}, \Psi_{R}\right) \in(\mathbf{1 0}, \overline{\mathbf{1 0}})$, with invariant $\epsilon^{i j k m n}\left(\mathbf{1 0}_{R}\right)_{i j}\left(\mathbf{1 0}_{L}\right)_{k m}\left(\mathbf{5}_{\Sigma}\right)_{n}$. Since $b_{L}=\left(\mathbf{1 0}_{L}\right)_{54}$ and $b_{R}=\left(\overline{\mathbf{1 0}}_{R}\right)_{34 / 43}$, a mass term $\bar{b}_{R} b_{L}$ is forbidden and the bottom mass can only be generated at higher order, explaining in a natural way the bottom/top mass gap. 


\begin{tabular}{|c|cc|}
\hline & $\mathbf{5}$ & $\mathbf{1 0}$ \\
\hline$g_{q_{L}}$ & $\left|u_{3}\right|^{2}+\left|u_{4}\right|^{2},\left|u_{1}\right|^{2}+\left|u_{2}\right|^{2}$ & $\left(\begin{array}{cc}\left|u_{5}\right|^{2}+\left|u_{3}\right|^{2} & u_{4}^{*} u_{3} \\
u_{4} u_{3}^{*} & \left|u_{5}\right|^{2}+\left|u_{4}\right|^{2}\end{array}\right)$ \\
$g_{t_{R}}$ & $\left|u_{5}\right|^{2}$ & $\left|u_{1}\right|^{2}+\left|u_{2}\right|^{2}+\left|u_{3}\right|^{2}+\left|u_{4}\right|^{2}$ \\
$g_{b_{R}}$ & $\left|u_{5}\right|^{2}$ & $\left|u_{3}\right|^{2}+\left|u_{4}\right|^{2}$ \\
$g_{t q}$ & $u_{5}\left(\begin{array}{c}u_{3} \\
u_{4}\end{array}\right)^{\dagger}$ & $u_{5}^{*}\left(\begin{array}{c}u_{1} \\
u_{2}\end{array}\right)^{T}\left(i \sigma_{2}\right)$ \\
$g_{b q}$ & $u_{5}\left(\begin{array}{c}u_{1} \\
u_{2},\end{array}\right)^{\dagger}$ & $u_{5}^{*}\left(\begin{array}{c}u_{3} \\
u_{4}\end{array}\right)^{T}\left(i \sigma_{2}\right)$ \\
\hline
\end{tabular}

Table 2. Couplings between the SM fermions and the $u$ variables, as defined in eq. (4.1), according to the $\mathrm{SU}(5)$ representation in which they are embedded. The result can be applied both to $\mathrm{SU}(5) / \mathrm{SU}(4)$ and $\mathrm{SU}(5) / \mathrm{SU}(4) \times \mathrm{U}(1)$ choosing the appropriate expression for $u$. In the case of the 5 , the first value for $g_{q_{L}}$ refers to $q_{L}^{t}$, the second to $q_{L}^{b}$.

However in both this cases there is no direct couplings between the fermions and the $u_{5}$.

(c) $\left(\Psi_{L}, \Psi_{R}\right) \in(\mathbf{5}, \overline{\mathbf{5}})$, which leads to a phenomenology similar to the $(\mathbf{5}, \mathbf{5})$ case up to a phase in the Yukawa interactions.

Let us comment on how the flavor problem is solved in our case. As pointed out in [11], the problem is twofold: on the one hand, one has to face the usual 2HDM problem of ensuring Minimal Flavor Violation (MFV) in the Yukawa sector; on the other hand, there could be more invariant Yukawa couplings of the fermions to the Higgs n.l.r. and a priori there is no reason to have alignment between the different flavor matrices defining different couplings [16]. In the $\mathrm{SU}(5)$ models under consideration, the flavour mixing is solely given by the mass matrices so that MFV is realized because

(i) the representations considered, $\mathbf{5}$ and $\mathbf{1 0}$, are small enough to allow only one embedding of the up and down quarks, as displayed in eq. (4.2);

(ii) all the fermions only couple to one $\Sigma$, so that their couplings to the Higgs are flavour diagonal.

Hence, there is no tree-level flavour changing from the Higgs sector. Unfortunately, the $t$-quark, instrumental in EWSB, couples to $u_{3}$, which is a combination of the real part of a Higgs field and the imaginary part of the other, pushing for the misalignment for their vev's. To point out this serious problem, we briefly discuss the PNGB potential in the next section. 


\section{Scalar potential}

The Higgs fields arise as Goldstone modes, so the shift symmetry forbids a tree level potential. However, the global symmetry is explicitly broken once the SM group is gauged and the fermions are coupled to the Higgs system, so that the Goldstone modes get a Coleman-Weinberg potential. The potential can be computed with standard techniques, and may be written in general as

$$
V=V_{\text {gauge }}+V_{\text {fermion }}
$$

In terms of the $u$ variables, and to lowest order in $g^{2}$ and $g^{\prime 2}$, the gauge part of the potential for the neutral components reads

$$
V_{\text {gauge }}=\frac{3}{2} \int \frac{d^{2} Q}{16 \pi^{2}} \frac{\Pi_{1}}{\Pi_{0}}\left(\frac{3 g^{2}+g^{\prime 2}}{4}\left(\left|u_{2}\right|^{2}+\left|u_{3}\right|^{2}\right)+D^{A} D^{A}\right)
$$

with $D^{A}=g_{A} u^{\dagger} T^{A} u$. This term is the additional contribution arising from the terms quartic in $u$ in the gauge part of the Lagrangian, as given in eq. (2.9), and closely resembles the supersymmetric case (without $B \mu$ term).

The fermionic contribution $V_{\text {fermion }}$ in eq. (5.1) can be simplified in the reasonable limit in which $b_{R}$ has a relatively small coupling to the strong sector. With this assumption, and to lowest order in the ratios of structure functions $\Pi_{1}^{a} / \Pi_{0}^{a}$, defined in eq. (4.1)) the potential for the neutral components results:

$$
\begin{aligned}
V_{\text {fermion }}^{(\mathbf{1 0})}=\int \frac{d^{4} Q}{16 \pi^{2}}[ & \left(\rho^{q_{3}}+\frac{1}{2} \rho^{t_{R}}\right)\left(\left|u_{2}\right|^{2}+\left|u_{3}\right|^{2}\right)+\left(\rho^{q_{3}}-\frac{f^{2}}{Q^{2}} Y_{t}^{2}\right)\left|u_{3}\right|^{2} \\
& \left.+\frac{1}{2} \rho^{t_{R}}\left(\left|u_{2}\right|^{2}+\left|u_{3}\right|^{2}\right)^{2}+\frac{f^{2}}{Q^{2}} Y_{t}^{2}\left(\left|u_{2}\right|^{2}+\left|u_{3}\right|^{2}\right)\left|u_{3}\right|^{2}+\ldots\right]
\end{aligned}
$$

for fermions embedded in a $\mathbf{1 0}$, and

$$
\begin{aligned}
V_{\text {fermion }}^{(\mathbf{5})}=\int \frac{d^{4} Q}{16 \pi^{2}}[ & \rho^{q_{3}}\left(1-\left|u_{2}\right|^{2}-\left|u_{3}\right|^{2}\right)+\rho^{t_{R}}\left(\left|u_{2}\right|^{2}+\left|u_{3}\right|^{2}\right) \\
& \left.-\frac{f^{2}}{Q^{2}} Y_{t}^{2}\left|u_{3}\right|^{2}\left(1-\left|u_{2}\right|^{2}-\left|u_{3}\right|^{2}\right)+\rho^{t_{R}}\left|u_{2}\right|^{2}\left|u_{3}\right|^{2}+\ldots\right]
\end{aligned}
$$

for fermions embedded in a $\mathbf{5}$, where the integrands are the ratios,

$$
\rho^{f}\left(Q^{2}\right)=\frac{\Pi_{1}^{f}}{\Pi_{0}^{f}},\left(f=t_{R}, b_{R}, q_{3}\right) \quad \text { and } \quad Y_{t}\left(Q^{2}\right)=\frac{\left|M_{1}^{t}\right|^{2}}{\Pi_{0}^{q_{3}} \Pi_{0}^{t_{R}}}
$$

Of course, a quantitative discussion of the Higgs vev and the spectrum is pointless in presence of so many unknown form factors. However, some comments are now in order:

1. To return to the Higgs alignment issue, notice that the important negative contribution in the potential corresponds to the $t$-quark Yukawa coupling to $u_{3}$. Hence it drives the vacuum through the $u_{3}$ direction which breaks the custodial symmetry as 
already stressed. The $b$-quark coupling to $u_{2}$, neglected in (5.3), (5.4), is too small to compete. As already quoted above, Higgs alignment can be enforced in composite $2 \mathrm{HDM}$ by discrete symmetries [11] that allow to restrict the couplings of SM fermions to a single Higgs in the case of the $\mathrm{SU}(4) / \mathrm{SU}(2) \times \mathrm{SU}(2) \times \mathrm{U}(1)$ model. In our case, the interesting property of the models is precisely the automatic uniqueness of these couplings and also the fact that quarks of different charges interact with different Higgs fields. Therefore, we cannot impose a symmetry that, at most, would kill our single Yukawa coupling. We have to rely on a partial compensation mechanism.

2. Since the custodial symmetry is essentially broken by the Higgs coupling to $q_{L}$ (see appendix A) that explicitly breaks $\mathrm{SU}(2)_{R}$, one could try to improve the situation introducing custodial fermions in the $(\mathbf{2 , 2})$ of $\mathrm{SU}(2)_{L} \times \mathrm{SU}(2)_{R}[21]$ to alleviate the constraint from the $T$-parameter. Interestingly, they might also contribute through loops to increase $B R(h \rightarrow \gamma \gamma)$ [22], in agreement with the recent observations at the LHC. They naturally provide an interesting phenomenological set up, detailed study of which is beyond the mandate of this paper.

3. For $\mathrm{SU}(5) / \mathrm{SU}(4)$, the $\varphi_{0}$ component of $u$ is a main concern too, since it is the PNGB associated to a abelian symmetry and, as such, an axion-like scalar subject to strict experimental bounds.It always appears in the potential as $\left|u_{5}\right|^{2}=$ $1-\sin ^{2}\left(\frac{\varphi}{f}\right) \frac{|\Phi|^{2}+|\tilde{\Phi}|^{2}}{\varphi^{2}}$ The fact that $\varphi_{0}$ only appears as a function of $\varphi$ implies that there is always a massless particle in the spectrum. Indeed, if we consider the simplified case in which $u$ has only one non vanishing component, since $V=V\left(|u|^{2}\right)$, at the minimum the mass matrix in the $\left(u, \varphi_{0}\right)$ basis has the form

$$
M^{2}=\left(\begin{array}{cc}
1 & \frac{\partial|u|^{2}}{\partial \eta} \\
\frac{\partial|u|^{2}}{\partial \eta} & \left(\frac{\partial|u|^{2}}{\partial \eta}\right)^{2}
\end{array}\right) \partial^{2} V / \partial|u|^{2}
$$

and has a zero eigenvalue. The result trivially generalizes to more $u$ components. This problem is independent of the choice of fermion embeddings. It may be solved introducing two or more right handed neutrinos. Consider $N_{R}^{1,2}$, two neutrino that couple to two different strong sector operators, having representations $N_{R}^{1,2}=5+$ $\zeta^{1,2} \overline{5}$. In this case a potential for the singlet field is generated at the leading order by the coupling $N_{R}^{1} \bar{N}_{R}^{2} \Sigma+h . c$. . This might lead to interesting phenomenological consequences $^{4}$ that are beyond the scope of the present paper.

\section{Other composite 2HDM's: non-linear realizations, PNGB Lagrangian and $T$ parameter}

As pointed out in the previous section, the presence of a term quartic in $u$ in the metric generates a contribution to the $T$ parameter already at tree level. This despite the presence of a large residual global symmetry $H \subset G$ containing the custodial symmetry as a subgroup.

\footnotetext{
${ }^{4}$ In particular, the breaking of lepton number by the strong sector should be treated carefully [12]. We thank J. Serra for calling our attention on this point.
} 
In this section we display the PNGB Lagrangian for the different cosets leading to 2HDM's. We consider cosets $G / H_{1} \times H_{2}$ with $H_{2}=\emptyset, \mathrm{U}(1), \mathrm{SU}(2)$ and we generalize the approach in section 2. As already noticed above for $G=\mathrm{SU}(5)$, it is useful to introduce as coset coordinates $p$ orthogonal unit vectors $u^{\alpha}(\alpha=1, \ldots, p)$ in the fundamental representation $\mathbf{N}$ of $G$ whose scalar products satisfy:

$$
u_{\alpha}^{\dagger} u^{\beta}=\delta_{\alpha}^{\beta} \quad \operatorname{tr} u^{\dagger} u \equiv \sum_{\alpha} u_{\alpha}^{\dagger} u^{\alpha}=p
$$

where $p=1$ for $H_{2}=\emptyset, \mathrm{U}(1)$ and $p=2$ for $H_{2}=O(2), \mathrm{SU}(2)$. They are functions of the PNGB fields, $\xi^{A}$ defined by

$$
u(\xi)=e^{i \Pi(\xi)} u(0) \quad \Pi(\xi)=\sum_{A} \xi_{A} T^{A}
$$

where $\xi_{A}$ are the PNGB fields and $T^{A}$ are the coset charges, while $u(0)$ corresponds to the vev('s) of one or more n.l.r. of the Higgs in fundamental representation(s) needed for the breaking. $G$ acts as $u \rightarrow g u h_{2}^{\dagger}(g, \xi)$. In particular, as already displayed above for $G=\mathrm{SU}(5)$, n.l.r. transforming as larger irreducible transformations are obtained with $\Sigma=u u^{\dagger}$ for the adjoint representation, $\Sigma=u u^{T}$ for the symmetric, etc. Notice the contraction of the $p$ indices associated to the action of $\mathrm{H}_{2}$.

For $G=\mathrm{SU}(5)$ the results are already given in (2.9). For the other cosets associated to C2HDM's, we just display the PNGB Lagrangians that are obtained along the same lines. For simplicity, the Lagrangians are arbitrarily normalized. ${ }^{5}$ There are two invariants with two derivatives, one quadratic, and the other quartic in the $u$ 's. By replacing $U^{\dagger} \partial_{\mu} U=$ $i d_{\mu}^{i} T^{i}+i E_{\mu}^{a} T^{a}$ as explained in section 2, we recall that by $H_{1}$ invariance of $u(0)$, the quadratic term becomes $\sum d_{\mu}^{i 2}+c \sum^{\prime} E_{\mu}^{a 2}$, with $\sum^{\prime}$ restricted to the $H_{2}$ charges, while only the last term is present in the quartic term (with another coefficient). By the appropriate combination of the two invariants, we thus obtain the CCWZ Lagrangian, $\sum d_{\mu}^{i 2}$, in terms of the $u$ 's.

First, consider $\mathrm{SO}(6) / \mathrm{SO}(4) \times \mathrm{SO}(2) \equiv \mathrm{SU}(4) / \mathrm{SU}(2) \times \mathrm{SU}(2) \times \mathrm{U}(1)$ and let us first consider the construction in terms of two 4 representations of $\mathrm{SU}(4)$ satisfying the previous rules, namely, $u_{i}^{\alpha}(\alpha=1,2 ; i=1, \ldots, 4), H_{2}=\mathrm{SU}(2)$. Then,

$$
\mathcal{L}_{\mathrm{PNGB}}=f^{2} \operatorname{tr}\left(\partial^{\mu} u^{\dagger} \partial_{\mu} u-u^{\dagger} \partial_{\mu} u \partial^{\mu} u^{\dagger} u\right) \quad\left(\mathrm{SU}(4) / \mathrm{SU}(2)^{2} \times \mathrm{U}(1)\right)
$$

But, this can be also written in terms of two (real) vectors of $\mathrm{SO}(6)$, denoted $v_{a}^{\alpha}(\alpha=$ $1,2 ; a=1, \ldots, 6), H_{2}=\mathrm{SO}(2)$ to obtain,

$$
\mathcal{L}_{\mathrm{PNGB}}=f^{2} \operatorname{tr}\left(\partial^{\mu} v^{T} \partial_{\mu} v-v^{T} \partial_{\mu} v \partial^{\mu} v^{T} v\right) \quad(\mathrm{SO}(6) / \mathrm{SO}(4) \times \mathrm{SO}(2))
$$

which, up to a normalization, is the same as (6.3) if one replaces $v_{a}^{\alpha}=\bar{u}^{\alpha} \Gamma_{a} u^{\alpha}$, where $\Gamma_{a}$ are $\mathrm{SO}(6)$ Dirac matrices.

\footnotetext{
${ }^{5}$ Actually the normalization can be fixed the end, having fitted to data the expressions for the gauge boson masses and normalized the Higgs boson kinetic term.
} 
The $\mathrm{Sp}(6) / \mathrm{Sp}(4) \times \mathrm{SU}(2)$ model has some interesting properties. With $H_{1}=\mathrm{Sp}(4)$ and $H_{2}=\mathrm{SU}(2)$, the n.l.r. can be displayed in terms of two 6-vectors such that $u_{2}=\Omega u_{1}^{*}$ leading to a triplet of quaternions as discussed in appendix $\mathrm{D}$. With the procedure described above we get

$$
\mathcal{L}=f^{2} \operatorname{tr}\left(\partial^{\mu} u^{\dagger} \partial_{\mu} u-\partial^{\mu} u^{\dagger} u u^{\dagger} \partial_{\mu} u\right) \quad(\operatorname{Sp}(6) / \operatorname{Sp}(4) \times \mathrm{SU}(2))
$$

The $\mathrm{SO}(9) / \mathrm{SO}(8)$ manifold is the 8-sphere, resulting in the corresponding metric for the Lagrangian for the real 9-vector $u$,

$$
\mathcal{L}_{\mathrm{PNGB}}=f^{2} \operatorname{tr}\left(\partial^{\mu} u^{T} \partial_{\mu} u\right) \quad(\mathrm{SO}(9) / \mathrm{SO}(8))
$$

Here, $H_{1}=\mathrm{SO}(8)$ and, since there is no $H_{2}$, the quadratic terms already contains only the PNGB part.

The next step is to define the embedding of the SM charges inside $H_{1} \times H_{2}$. Then, substituting the standard derivatives with the SM covariant derivatives, $\partial u_{i} \rightarrow \partial u_{i}-$ $i(\hat{W} u)_{i}$, with $\hat{W}_{\mu}=g W_{\mu}^{+} T_{L}^{-}+g W_{\mu}^{+} T_{L}^{-}+e A_{\mu}\left(T_{L}^{3}+T_{R}^{3}\right)+g \sec \theta_{W} Z_{\mu}\left(T_{L}^{3}-\tan ^{2} \theta_{W} T_{R}^{3}\right)$, the gauge boson masses and possible contributions to the $T$ parameter can be read from the previous expressions. All 2HDM's have $\mathrm{SU}(2)_{L} \times \mathrm{SU}(2)_{R}$ as a subgroup of the linear symmetry of the coset with the Higgs fields transforming in two $(\mathbf{2}, \mathbf{2})$, so to define the wanted custodial symmetry. Let us take first $\mathrm{SU}(2)_{L} \times \mathrm{SU}(2)_{R} \subset H_{1}$. The mass matrix for the gauge bosons can be written,

$$
\frac{\mathcal{M}_{A B}^{2}}{g_{A} g_{B}}=u^{\dagger}\left\{T^{A}, T^{B}\right\} u-\kappa u^{\dagger} T^{A} u u^{\dagger} T^{B} u
$$

where $\kappa$ is given in the different $\mathcal{L}_{\mathrm{PNGB}}$ above, and where only the neutral components are retained, so that $\left(T_{L}^{3}+T_{R}^{3}\right) u=0$. Since all the states are doublets or singlets of each $\mathrm{SU}(2)$, $u^{\dagger}\left\{T^{A}, T^{B}\right\} u=1 / 2 \delta^{A B} \sin ^{2}(\varphi / f)$. The first term is analogous to the SM gauge bosons mass term. The second term is easily calculated, and gives the additional contribution to the $Z$-mass in (3.4) and the corresponding anomalous contrition to the $T$-parameter. Although the calculation is straightforward, we justify the results from a group theoretical point of view in appendix B.

Up to the factor $\kappa$ the result is the same for all models where the SM gauge symmetries are embedded in $H_{1}$ and that are strongly constrained by $T$ unless the Higgs are aligned. The exception are the models based on $\mathrm{SO}(9) / \mathrm{SO}(8)$ which have only the quadratic part. In view of this success it deserves some limelight and it is further analysed in the next section.

The $\mathrm{Sp}(6) / \mathrm{Sp}(4) \times \mathrm{SU}(2)$ models are special because, according to the embedding of the SM they can have enough symmetry to preserve the custodial symmetry that protects the $T$ parameter [11]. Indeed, with the Higgs bosons in the $(\mathbf{2 , 2})$, one of the factors in $\mathrm{SU}(2)_{L} \times \mathrm{SU}(2)_{R}$ must be in $H_{2}=\mathrm{SU}(2)$ and the other in $H_{1}=\mathrm{Sp}(4)$. The latter can come from a maximal subgroup $\mathrm{SU}(2) \times \mathrm{U}(1)$ of $\mathrm{Sp}(4)$ or from the $\mathrm{SU}(2) \times \mathrm{SU}(2)$ one. Only the latter has sufficient symmetry to protect $T$, since the two Higgs vev's become invariant under the $\mathrm{SU}(2)_{C}$ defined by the sums of the charges in the three $\mathrm{SU}(2)$ factors. This is further discussed in appendix B and the final result is

$$
M_{W}=M_{Z} \cos \left(\theta_{W}\right)=\frac{g f}{\sqrt{2}} \sin (h / f) \cos (h / f)
$$


where $h$ is the combined vev's of the two Higgs. Notice that higher order couplings of the Higgs sector to the gauge bosons are present at tree-level even if $\rho=1$ and, also, the factor $\cos (h / f)$ with respect to the other models.

\section{A composite two Higgs doublet model based on $\mathrm{SO}(9) / \mathrm{SO}(8)$}

We turn now to a composite $2 \mathrm{HDM}$ based on the symmetry breaking pattern $\mathrm{SO}(9) / \mathrm{SO}(8)$. As stressed in section 6 , there is no tree-level contribution to the $T$ parameter. The n.l.r. is a 9 -vector

$$
u=e^{\frac{i \Pi}{f}} u_{0}=\left(\begin{array}{c}
\frac{\sin (\varphi / f)}{\varphi} \phi \\
\cos (\varphi / f)
\end{array}\right)
$$

where $\varphi=\sqrt{\phi^{T} \phi}$, and $\phi$ the vector containing the eight NGBs belonging to a $\mathbf{8}_{\mathbf{V}}$ of $\mathrm{SO}(8) .{ }^{6}$ The PNGB Lagrangian has the single term in eq. (6.6). We need to embed the SM gauge group in $\mathrm{SU}(2) \times \mathrm{SU}(2) \subset \mathrm{SO}(8)$ to include the necessary custodial symmetry, and the two Higgses in the $\mathbf{8}_{\mathbf{V}}$ transform as two $(\mathbf{2}, \mathbf{2})$. The selection of these embeddings are given in appendix C. There are two inequivalent choices that basically differ by a $\mathrm{U}(1)$ factor that differentiates between the Higgs doublets:

$$
\begin{aligned}
& \mathbf{8}_{\mathrm{V}}=(\mathbf{2}, \mathbf{2})+(\mathbf{2}, \mathbf{2}) \in \mathrm{SU}(2) \times \mathrm{SU}(2) \subset \mathrm{SO}(8) \\
& \mathbf{8}_{\mathbf{V}}=(\mathbf{2}, \mathbf{2})_{+\mathbf{1}}+(\mathbf{2}, \mathbf{2})_{-\mathbf{1}} \in \mathrm{SU}(2) \times \mathrm{SU}(2) \times \mathrm{U}(1) \subset \mathrm{SO}(8)
\end{aligned}
$$

In the first case, the fermions can couple to both Higgs doublets, which is a source of flavour changing. We do not investigated possible discrete symmetries and concentrate on the second embedding where the Higgs doublets have opposite U(1) charges.

To guarantee a correct electroweak charge assignment for the Higgs doublets, the additional $\mathrm{U}(1)$ should not take part in the definition of the hypercharge, still given by $Y=T_{L}^{3}+T_{R}^{3}$. With these assignments, replacing the SM covariant derivative in eq. (6.6), one obtains the masses of the gauge bosons

$$
\mathcal{L}_{m}=\frac{1}{2} g^{2} f^{2} \sin ^{2}(\varphi / f)\left(W_{\mu}^{+} W_{\mu}^{-}+\frac{1}{2 \cos \theta_{W}} Z_{\mu} Z_{\mu}\right),
$$

so that there are no tree-level restriction on $v / f$, independently of the alignment between the two Higgs doublet in the vacuum.

Let us turn now to the fermions. Once the NGBs are assigned to the $\mathbf{8}_{\mathbf{V}}$ of $\mathrm{SO}(8)$ decomposing under $\mathrm{SU}(2) \times \mathrm{SU}(2) \times \mathrm{U}(1)$ as $(\mathbf{2}, \mathbf{2})_{+\mathbf{1}}+(\mathbf{2}, \mathbf{2})_{-\mathbf{1}}$, the other two eightdimensional representations of $\mathrm{SO}(8)$ transform under the $\mathrm{SU}(2) \times \mathrm{SU}(2) \times \mathrm{U}(1)$ subgroup as: $\boldsymbol{8}_{\mathrm{s}}=(\mathbf{1}, \mathbf{1})_{+\mathbf{2}}+(\mathbf{1}, \mathbf{1})_{-\mathbf{2}}+(\mathbf{3}, \mathbf{1})_{\mathbf{0}}+(\mathbf{1}, \mathbf{3})_{\mathbf{0}}$ and $\boldsymbol{8}_{\mathrm{c}}=(\mathbf{2}, \mathbf{2})_{-\mathbf{1}}+(\mathbf{2}, \mathbf{2})_{+\mathbf{1}}$. The assignments for $\mathrm{U}(1)_{X}$ are parallel to those in section 4 . Barring larger representations, there are two choices for the fermions, which are made explicit below together with, in brackets, their embeddings in $\mathrm{SO}(9) \times \mathrm{U}(1)_{X}$ :

\footnotetext{
${ }^{6}$ We use here the conventions for the $\mathrm{SO}(8)$ representations and subgroups from [24]
} 
1. $t_{L} \in \mathbf{8}_{\mathbf{V}}\left(\in \mathbf{9}_{2 / 3}\right), b_{L} \in \mathbf{8}_{\mathbf{V}}\left(\in \mathbf{9}_{-1 / 3}\right), b_{R} \in \mathbf{1}\left(\in \mathbf{1}_{-1 / 3}\right), t_{R} \in \mathbf{1}\left(\in \mathbf{1}_{2 / 3}\right)$. A brane mass term allows for the reconstruction of the $q_{L}$ doublet as sketched in section 4 . The Yukawa couplings are the singlets in the products $\mathbf{8}_{\mathrm{V}} \times \mathbf{8}_{\mathrm{V}} \times \mathbf{1} \in \mathbf{9} \times \mathbf{9} \times \mathbf{1}$.

2. $q_{L} \in \mathbf{8}_{\mathbf{c}}\left(\in \mathbf{9}_{2 / 3}\right), t_{R}, b_{R} \in \mathbf{8}_{\mathbf{s}}\left(\in \mathbf{8}_{\mathbf{s} 2 / 3}\right)$ so that all fermions transform under $\mathrm{SO}(9) \times$ $\mathrm{U}(1)_{X}$ as a $\mathbf{1 6}=\mathbf{8}_{\mathbf{s}}+\boldsymbol{8}_{\mathbf{c}}$ of $\mathrm{SO}(8)$, both $t_{R}$ and $b_{R}$ belonging to the $(\mathbf{1}, \mathbf{3})$ of $\mathrm{SU}(2) \times$ $\mathrm{SU}(2)$. The Yukawa invariants are in the products $\mathbf{8}_{\mathbf{V}} \times \mathbf{8}_{\mathbf{s}} \times \mathbf{8}_{\mathbf{c}} \in \mathbf{9} \times \mathbf{1 6} \times \mathbf{1 6}$.

The latter seems more attractive, in particular because it is naturally consistent with gauge-Higgs unification scenarios. In both cases, the fermions couple only to the Higgs doublet with opposite $\mathrm{U}(1) \subset \mathrm{SO}(8)$ charge, so that the other one is a so-called inert Higgs. ${ }^{7}$ Therefore the FCNC effects are suppressed because each fermion couples to only one and the same Higgs doublet and each fermion can be uniquely embedded in $\mathrm{SO}(8) \times \mathrm{U}(1)$ representations. The model satisfies all the items in our fermion shopping list at the beginning of section 4 .

\section{Conclusions}

In this paper we have studied some composite Higgs models with extended scalar sectors. A detailed analysis has been carried out for the cosets $\mathrm{SU}(5) / \mathrm{SU}(4) \times \mathrm{U}(1)$, and $\mathrm{SO}(9) / \mathrm{SO}(8)$, which deliver two SM doublets or, for SU(5)/SU(4), two SM doublets and one singlet.

In both the $\mathrm{SU}(5)$ cases, a contribution to the $T$ parameter is present already at tree level unless there is an unexpected alignment of vevs in the scalar potential. A survey of various C2HDM's shows that this is naturally expected in other examples of this class of models, unless the symmetries defining the coset are large enough so that any element in the coset is invariant under an $\mathrm{SU}(2)$, so defining the custodial symmetry. We observe that two specific cosets, $\mathrm{Sp}(6) / \mathrm{Sp}(4) \times \mathrm{SU}(2)$ and $\mathrm{SO}(9) / \mathrm{SO}(8)$ exhibit such a group theoretical property, hence no invariants contributing to $T$ can be written. This ensures an effective custodial symmetry at tree level independent from the scalar potential.

Turning to fermions, we analyzed the embedding of the matter fields in the smallest $\mathrm{SU}(5)$ and $\mathrm{SO}(9)$ representations, paying attention to the effects on the $Z b \bar{b}$ vertex and on the presence of Higgs-mediated FCNC. In the $\mathrm{SU}(5)$ cases, we find that $Z b \bar{b}$ is protected only when the SM fermions are embedded in a 10, while Higgs-mediated FCNC are always absent because the non linear Higgs has a single coupling to fermions. The up and down type quarks couple to different linear combinations of the Higgs doublets, so that the latter are both active. However the $t$ quark coupling tends to misalign the Higgs vev's and increases the contribution to $T$. Therefore, these $\mathrm{SU}(5)$ models do need a compensation mechanism to be phenomenologically viable.

In the $\mathrm{SO}(9)$ case an additional abelian charge embedded in $\mathrm{SO}(8)$ can be used to ensure the fermions to be coupled to just one of the doublets, making the other one inert. Moreover, when the left handed quark doublet is embedded in the spinorial representation, also the $Z b \bar{b}$ vertex is protected.

\footnotetext{
${ }^{7}$ For a recent discussion of inert Higgs phenomenology see [11].
} 


\section{Acknowledgments}

We thank J. Serra, M. Redi and A. Wulzer for useful comments. E.B. thanks M. Frigerio and R. Contino for useful discussions. This work has been partially supported by the Agence Nationale de la Recherche under contract ANR 2010 BLANC 041301. The work of TSR is supported by EU ITN, contract "UNILHC" PITN-GA-2009-237920 and the CEAEurotalents program. HS is supported by a CAPES Foundation (Ministry of Education of Brazil) Postdoc Fellowship.

\section{A Embedding of the Standard Model fermions in SU(5) representations}

The SM charges, embedded in the first four entries of the $5 \times 5 \mathrm{SU}(5)$, are given by

$$
T_{L}^{i}=1_{2 \times 2} \times \tau^{i}, \quad T_{R}^{3}=\tau^{3} \times 1_{2 \times 2}
$$

with $\tau^{i}$ the usual $\mathrm{SU}(2)$ generators. In terms of $\left(T_{L}^{3}, T_{R}^{3}\right)$ charges, this choice corresponds to a $\mathrm{SU}(5)$ fundamental decomposed as

$$
\mathbf{5}=\left(\begin{array}{c}
(+,+) \\
(-,+) \\
(+,-) \\
(-,-) \\
(0,0)
\end{array}\right) .
$$

The $\mathrm{SU}(5)$ fundamental decomposes as $\mathbf{5}=(\mathbf{2}, \mathbf{2})+(\mathbf{1}, \mathbf{1})$ under $\mathrm{SU}(2)_{L} \times \mathrm{SU}(2)_{R}$. For $\mathrm{SU}(5) / \mathrm{SU}(4)$, the two LH quarks must be embedded in different bidoublets:

$$
\begin{array}{rlr}
q_{L}^{t} & \in(\mathbf{2}, \mathbf{2})_{\mathbf{2} / \mathbf{3}} & \text { with }_{L}=(+,-) \\
q_{L}^{b} & \in(\mathbf{2}, \mathbf{2})_{-\mathbf{1} / \mathbf{3}} & \text { with }_{L}=(-,+) \\
u_{R} & \in(\mathbf{1}, \mathbf{1})_{\mathbf{2} / \mathbf{3}} & \\
d_{R} & \in(\mathbf{1}, \mathbf{1})_{-\mathbf{1} / \mathbf{3}} &
\end{array}
$$

where in the last column we specified the $\left(T_{L}^{3}, T_{R}^{3}\right)$ assignment.

The specific embeddings are

$$
\Psi_{q_{L}^{t}}=\left(\begin{array}{c}
0_{2 \times 1} \\
q_{L} \\
0
\end{array}\right)_{2 / 3} \Psi_{q_{L}^{b}}=\left(\begin{array}{c}
q_{L} \\
0_{2 \times 1} \\
0
\end{array}\right)_{-1 / 3} \Psi_{t_{R}}=\left(\begin{array}{c}
0_{2 \times 1} \\
0_{2 \times 1} \\
t_{R}
\end{array}\right)_{2 / 3} \Psi_{b_{R}}=\left(\begin{array}{c}
0_{2 \times 1} \\
0_{2 \times 1} \\
b_{R}
\end{array}\right)_{-1 / 3}
$$

Turning to the $\mathrm{SU}(5)$ symmetric representation, it corresponds to $\mathbf{1 0}=(\mathbf{2}, \mathbf{2})+(\mathbf{1}, \mathbf{3})+$ $(\mathbf{3}, \mathbf{1})$ under $\mathrm{SU}(2)_{L} \times \mathrm{SU}(2)_{R}$. To accommodate hypercharge, the $\mathrm{U}(1)_{X}$ charge has to be fixed to $X=2 / 3$, with assignment

$$
\begin{array}{rll}
q_{L} \in(\mathbf{2}, \mathbf{2})_{\mathbf{2} / \mathbf{3}} & \text { with } & t_{L}=(+,-), b_{L}=(-,-) \\
u_{R} \in(\mathbf{1}, \mathbf{3})_{\mathbf{2} / \mathbf{3}} & \text { with } & t_{R}=(0,0) \\
d_{R} \in(\mathbf{1}, \mathbf{3})_{\mathbf{2} / \mathbf{3}} & \text { with } & b_{R}=(0,-1)
\end{array}
$$


The explicit embeddings are

$$
\begin{aligned}
& \Psi_{q_{L}}=\frac{1}{2}\left(\begin{array}{c|c}
0_{4 \times 4} & 0_{2 \times 1} \\
& q_{L} \\
\hline 0_{1 \times 2} q_{L}^{T} & 0
\end{array}\right), \\
& \Psi_{t_{R}}=\frac{t_{R}}{2}\left(\begin{array}{cc|c}
0_{2 \times 2} & i \sigma_{2} & 0_{2 \times 1} \\
i \sigma_{2} & 0_{2 \times 2} & 0_{2 \times 1} \\
\hline 0_{1 \times 2} & 0_{1 \times 2} & 0
\end{array}\right), \\
& \Psi_{d_{R}}=\frac{b_{R}}{\sqrt{2}}\left(\begin{array}{cc|c}
0_{2 \times 2} & 0_{2 \times 2} & 0_{2 \times 1} \\
0_{2 \times 2} & i \sigma_{2} & 0_{2 \times 1} \\
\hline 0_{1 \times 2} & 0_{1 \times 2} & 0
\end{array}\right) .
\end{aligned}
$$

\section{B Algebraic analysis of the $T$-parameter}

In this appendix we give a more detailed analysis of the terms corresponding to anomalous $T$ parameters. The so-called $D$-terms, $D^{A}=u^{\dagger} T^{A} u$, where $T^{A}$ are the generators of a group $\mathrm{G}$ in the (arbitrary) representation of $u$, have been studied in the context of supersymmetry. For completeness, this appendix contains a the study of the anomalous $T$ parameter defined in (6.7) as $D^{A} D_{A}$, based on [25], from where we borrow the following lemma.

Given $u$ defined by its little group $H \subset G, D_{A}=u^{\dagger} T^{A} u \neq 0$, if and only if $T^{A} \in G / H$ and commutes with the elements of $H$.

This lemma has been very useful in the study of symmetry breaking in supersymmetric theories where the fields are complex, even when they transform in real representations if they belong to chiral supermultiplets. Here it is just a curiosity since the calculations are straightforward.

To apply it in our case, let us take $G=\mathrm{SU}(2) \times \mathrm{SU}(2)$ and $u \in(\mathbf{2}, \mathbf{2})$, and write it as a vector $u_{i}=x_{i}+y_{i},(i=1, \ldots, 4)$ such that the generators are real, antisymmetric and act on $\mathrm{u}$ as a 4-rotation, $T^{i j} u_{k}=\delta^{j k} u_{i}-\delta^{i k} u_{j}$. Notice that any $u$ can be rotated in the plane $u_{1}=u_{2}=0$, with little group $\mathrm{H}=\mathrm{U}(1)$ generated by the (electric) charge, $T^{12}$. Then $T^{34}$ is the only charge in the coset $\mathrm{G} / \mathrm{H}$ that commutes with $T^{12}$. Indeed, $u^{\dagger} T^{34} u=i\left(y_{3} x_{4}-y_{4} x_{3}\right)$ is the only non-vanishing component of the $D$-terms and, in this basis, $T^{34}$ is the charge corresponding to $Z$ and the anomalous contribution to the $Z$ mass is proportional to $\left(D^{34}\right)^{2}$.

Now consider a critical $u$, such that $x_{i}$ and $y_{i}$ are aligned and can be rotated to $u_{1}=u_{2}=u_{3}=0$. Its little group is the vectorial (or custodial) $H=\mathrm{SU}(2)$ of rotations in this hyperplane. The coset $\mathrm{G} / \mathrm{H}$ is given by the three axial charges that transform as a triplet under $\mathrm{H}$. Therefore all $D^{i j}=0$ and there is no anomalous term in the $Z$ mass. The conclusion is that the complex character of the $u$ coordinates, related to $H_{2}=\mathrm{U}(1)$ in the cosets defining the Higgs doublet as PNGB, is at the origin of the anomalous $T$ parameter in these cases, unless one can ensure the two Higgs alignment.

The case where $\mathrm{SU}(2) \times \mathrm{SU}(2)$ is embedded in both $H_{1}$ and $H_{2}$, is different since now the equivalent of the D-terms $D^{A}=u^{\dagger} T^{A} u$, are matrices. Let us concentrate on the relevant instance of $\mathrm{Sp}(6) / \mathrm{Sp}(4) \times \mathrm{SU}(2)$ and choose the embedding of the SM subgroup through 
the $\mathrm{SU}(2)^{2} \in \mathrm{Sp}(4)$, hence $\mathrm{SU}(2)^{3} \in \mathrm{Sp}(6)$, so that $u$ decomposes as $(\mathbf{2}, \mathbf{1}, \mathbf{2})+(\mathbf{1}, \mathbf{2}, \mathbf{2})$ while the bottom components are invariant. Due to the large symmetry, the two Higgs in $u$ can be aligned so that the generic $u$ encoding the two Higgs PNGB's has a little group the custodial group defined, with some convention, as the sum of the three $\mathrm{SU}(2)$ charges, defining the custodial $\mathrm{SU}(2)_{C}$. Therefore, the $T$ parameter must be non anomalous.

The two Higgs behave as $(\mathbf{2}, \mathbf{2})$ if we choose $\mathrm{SU}(2)_{L} \in$ the sum of those in $H_{1}=$ $\mathrm{Sp}(4)$ and $\mathrm{SU}(2)_{R}$ as the remaining one in $H_{2}$, or vice-versa. We recall that the latter 'acts from the right'. After the gauging of the SM symmetries one immediately finds the masses of the gauge bosons by rotating the Higgs quaternions in $u$ to the element $u^{T}=$ $\left(u_{0} \sin \beta \mathbf{1}_{2}, u_{0} \cos \beta \mathbf{1}_{2}, \cos (h / f) \mathbf{1}_{2}\right), u_{0}=h \sin (h / f)$, and $h$ is the usual combination of the Higgs vev's. The result,

$$
\frac{f^{2}}{2}\left(g^{2} W_{\mu}^{+} W^{-\mu}+\left(g W_{\mu}^{0}-g^{\prime} B_{\mu}\right)^{2}\right) \sin ^{2}(h / f) \cos ^{2}(h / f),
$$

where the term in $\sin ^{4}(h / f)$ comes from the quartic term in the PNGB lagrangian, is clearly consistent with the custodial symmetry, introducing a correction factor $\cos ^{2}(h / f)$ with respect to the results in the other $2 \mathrm{HDM}$ cosets.

\section{Embedding of the Standard Model charges in $\mathrm{SO}(9)$}

In this appendix we describe the embeddings of the $\mathrm{SO}(9) / \mathrm{SO}(8)$ coset $(\mathrm{PNGB}) \mathbf{8}_{\mathbf{V}}=$ $(\mathbf{2}, \mathbf{2})+(\mathbf{2}, \mathbf{2}) \in \mathrm{SU}(2) \times \mathrm{SU}(2) \subset \mathrm{SO}(8)$. A systematic way to find them is to follow all the chains of maximal subgroups starting from those of $\mathrm{SO}(8)$ down to those that contain $\mathrm{SU}(2) \times \mathrm{SU}(2)$ and discard all those that lead to the wrong decomposition for the $\mathbf{8} \mathbf{V}$ of PNGB. We follow the conventions in [24] and the results are easily checked form the tables therein. We simplify the notations by writing $\mathrm{SU}(2)^{n}$ for the $\times$ product of $n \mathrm{SU}(2)$ 's.

Two of the so-defined chains, namely $\mathrm{SO}(8) \rightarrow \mathrm{SU}(4) \times \mathrm{U}(1) \rightarrow \mathrm{SU}(2)^{2} \times \mathrm{U}(1)$ and $\mathrm{SO}(8) \rightarrow \mathrm{Sp}(4) \times \mathrm{SU}(2) \rightarrow \mathrm{SU}(2)^{2} \times \mathrm{U}(1)$, yield the decomposition:

$$
\begin{aligned}
8_{\mathrm{V}} & =(2,2)_{+1}+(2,2)_{-1} \quad 8_{\mathrm{c}}=(2,2)_{-1}+(2,2)_{+1} \\
\mathbf{8}_{\mathrm{s}} & =(\mathbf{1}, \mathbf{1})_{+2}+(\mathbf{1}, \mathbf{1})_{-2}+(3,1)_{0}+(\mathbf{1}, 3)_{0}
\end{aligned}
$$

where the $\mathrm{U}(1)$ charges are also displayed for each $\mathrm{SU}(2)^{2}$ representation.

Three other chains, namely, $\mathrm{SO}(8) \rightarrow \mathrm{SO}(7) \rightarrow \mathrm{SU}(4) \rightarrow \mathrm{SU}(2)^{2}, \mathrm{SO}(8) \rightarrow \mathrm{SO}(7) \rightarrow \mathrm{SU}(2)^{3}$ $\rightarrow \mathrm{SU}(2)^{2}$ and $\mathrm{SO}(8) \rightarrow \mathrm{SU}(2)^{4} \rightarrow \mathrm{SU}(2)^{2}$, have the same $\mathrm{SU}(2)^{2}$ decompositions, but since there is no $\mathrm{U}(1)$ factor, there is no distinction between the two $(\mathbf{2}, \mathbf{2})$ 's or the two $(\mathbf{1}, \mathbf{1})$ 's.

Finally, note that besides the quadratic invariant for each $\mathbf{8}$, there is the invariant $\mathbf{8}_{\mathrm{s}} \mathbf{8}_{\mathbf{V}} \mathbf{8}_{\mathbf{c}}$ that will be useful in the analysis of Yukawa couplings.

\section{Analysis of the $\mathrm{Sp}(6) / \mathrm{Sp}(4) \times \mathrm{SU}(2)$ coset}

$\operatorname{Sp}(6)$ is the group of unitary transformations such that $\Omega \equiv 1_{3} \times \sigma_{2}$ is invariant , i.e., $U \Omega U^{T}=\Omega$. It is useful to work in terms of quaternions: $Q=q^{\alpha} e_{\alpha}$, with $e_{i}=i \sigma_{i}, e_{4}=1_{2}$ Notice that: $Q^{\dagger} Q=\sum q^{i 2} 1_{2}, \sigma_{2} Q=Q^{*} \sigma_{2}$. Then, the $\operatorname{Sp}(6)$ algebra can be written as the 
$3 \times 3$ hermitian quaternion matrices with elements $i Q_{i j}$ such that $i Q_{i i}^{4}=0$. The diagonal elements $i Q_{i i},(i=1,2,3)$ are then associated with the $\mathrm{SU}(2)^{3}$ sub algebra. Adding only $i Q_{12}$ it is upgraded to $\mathrm{Sp}(4) \times \mathrm{SU}(2)$, while $i Q_{13}$ and $i Q_{23}$ define the coset $\mathrm{Sp}(6) / \mathrm{Sp}(4) \times \mathrm{SU}(2)$, transforming as a $(\mathbf{4 , 2})$ of $\mathrm{Sp}(4) \times \mathrm{SU}(2)$. In particular, the associated PNGB matrix $\Pi$ can be written as

$$
\Pi=\left(\begin{array}{c|c}
\mathbf{0}_{4} & -i \mathbf{H}_{1} \\
& -i \mathbf{H}_{2} \\
\hline i \mathbf{H}_{1}^{\dagger} i \mathbf{H}_{2}^{\dagger} & \mathbf{0}_{2}
\end{array}\right)
$$

where the $\mathbf{H}_{i} \equiv h_{i}^{\alpha} e_{\alpha}$ are the two Higgs doublets reshuffled into the familiar quaternion form.

We then define the vector with quaternion components $u_{i}, \quad(i=1,2,3)$ such that:

$$
u=e^{i \Pi\left(\mathbf{H}_{i}\right)} u(0)=\frac{\sin (h / f)}{h}\left(\begin{array}{c}
\mathbf{H}_{1} \\
\mathbf{H}_{2} \\
\cot (h / f) \mathbf{1}
\end{array}\right) \quad u(0)=\left(\begin{array}{c}
\mathbf{0}_{2} \\
\mathbf{0}_{2} \\
\mathbf{1}_{2}
\end{array}\right)
$$

where $h^{2}=\operatorname{tr} \sum_{i=1, \ldots, 4}\left(h_{1}^{i 2}+h_{2}^{i 2}\right)$ so that it transforms as defined in section 6, namely, $u \rightarrow g u h_{2}^{\dagger}\left(g, \mathbf{H}_{i}\right)$ with $g \in G$ and $h_{2} \in H_{2}$. Therefore $u$ can be also viewed as two orthogonal 6 -vectors $u_{\alpha}, \alpha=1,2$, each one transforming as the $\mathbf{6}$ of $\operatorname{Sp}(6)$, with $u_{2}=i \Omega u_{1}^{*}$. Notice that, as quaternions, $\Omega u=u^{*} \sigma_{2}$.

Another possible parametrization for the breaking $\mathrm{Sp}(6) \rightarrow \mathrm{Sp}(4) \times \mathrm{SU}(2)$ is given by the vacuum

$$
\Sigma_{0}=\left(\begin{array}{ccc}
\mathbf{0}_{2} & & \\
& \mathbf{0}_{2} & \\
& & \sigma_{2}
\end{array}\right),
$$

in an antisymmetric 14 of $\mathrm{Sp}(6)$. Then the n.l.r. can be written in terms of the PNBG as $\Sigma=U \Sigma_{0} U^{T}=u \sigma_{2} u^{T}$.

Following the procedure described in sections 2 and 6 to combine the invariants to have equivalence with the CCWZ construction, the kinetic Lagrangian results

$$
\mathcal{L}=\frac{f^{2}}{4}\left(\partial^{\mu} u^{\dagger} \partial_{\mu} u-\partial^{\mu} u^{\dagger} u u^{\dagger} \partial_{\mu} u\right)
$$

so that a tree-level contribution to $T$ is expected from the second term.

Using the $\mathrm{SU}(2)^{3}$ symmetry both quaternions $\mathbf{H}_{1}$ and $\mathbf{H}_{2}$ can be rotated and aligned along their components $e_{4}=\mathbf{1}_{2}$, which is invariant under a (diagonal) $\mathrm{SU}(2)$, the custodial symmetry. The effectiveness of custodial symmetry can also be explicitly checked substituting the ordinary derivatives in D.4 with covariant ones: $\partial_{\mu} u \rightarrow \partial_{\mu} u+i W_{\mu}^{a}\left(T_{L}^{a} u-u t_{L}^{a}\right)+$ $i B_{\mu} Y u$. In the notation of the previous sections, we are specializing to the case in which $\mathrm{U}(1)_{Y} \subset \mathrm{Sp}(4)$ and $\mathrm{SU}(2)_{L}=H_{2}$, so that the latter acts on $u$ both from the left and from the right. It is immediate to write the covariant derivative for the other case.

Turning to the fermions, the smaller $\mathrm{Sp}(6)$ representations decompose under $\mathrm{SU}(2)$ $\times \operatorname{Sp}(4)$ as

$$
\begin{aligned}
& \mathbf{1 4}=(\mathbf{2}, \mathbf{4})+(\mathbf{1}, \mathbf{5})+(\mathbf{1}, \mathbf{1}) \\
& \mathbf{2 1}=(\mathbf{2}, \mathbf{4})+(\mathbf{1}, \mathbf{1 0})+(\mathbf{3}, \mathbf{1})
\end{aligned}
$$


Since there are several ways to embed the SM fermions, a severe problem of Flavor Changing Neutral Currents is present.

Open Access. This article is distributed under the terms of the Creative Commons Attribution License which permits any use, distribution and reproduction in any medium, provided the original author(s) and source are credited.

\section{References}

[1] ATLAS collaboration, Observation of a new particle in the search for the standard model Higgs boson with the ATLAS detector at the LHC, Phys. Lett. B 716 (2012) 1 [arXiv: 1207.7214] [INSPIRE].

[2] CMS collaboration, Observation of a new boson at a mass of $125 \mathrm{GeV}$ with the CMS experiment at the LHC, Phys. Lett. B 716 (2012) 30 [arXiv:1207.7235] [INSPIRE].

[3] D.B. Kaplan and H. Georgi, $\mathrm{SU}(2) \times \mathrm{U}(1)$ breaking by vacuum misalignment, Phys. Lett. B 136 (1984) 183 [INSPIRE].

[4] H. Georgi, D.B. Kaplan and P. Galison, Calculation of the composite Higgs mass, Phys. Lett. B 143 (1984) 152 [INSPIRE].

[5] H. Georgi and D.B. Kaplan, Composite Higgs and custodial SU(2), Phys. Lett. B 145 (1984) 216 [inSPIRE].

[6] M.J. Dugan, H. Georgi and D.B. Kaplan, Anatomy of a composite Higgs model, Nucl. Phys. B 254 (1985) 299 [inSPIRE].

[7] K. Agashe, R. Contino and A. Pomarol, The minimal composite Higgs model, Nucl. Phys. B 719 (2005) 165 [hep-ph/0412089] [INSPIRE].

[8] R. Contino, L. Da Rold and A. Pomarol, Light custodians in natural composite Higgs models, Phys. Rev. D 75 (2007) 055014 [hep-ph/0612048] [INSPIRE].

[9] R. Contino, T. Kramer, M. Son and R. Sundrum, Warped/composite phenomenology simplified, JHEP 05 (2007) 074 [hep-ph/0612180] [INSPIRE].

[10] B. Gripaios, A. Pomarol, F. Riva and J. Serra, Beyond the minimal composite Higgs model, JHEP 04 (2009) 070 [arXiv:0902.1483] [INSPIRE].

[11] J. Mrazek, A. Pomarol, R. Rattazzi, M. Redi, J. Serra et al., The other natural two Higgs doublet model, Nucl. Phys. B 853 (2011) 1 [arXiv:1105.5403] [INSPIRE].

[12] M. Frigerio, J. Serra and A. Varagnolo, Composite GUTs: models and expectations at the LHC, JHEP 06 (2011) 029 [arXiv:1103.2997] [INSPIRE].

[13] S.L. Glashow and S. Weinberg, Natural conservation laws for neutral currents, Phys. Rev. D 15 (1977) 1958 [INSPIRE].

[14] A. Freitas and Y.-C. Huang, Electroweak two-loop corrections to $\sin ^{2}(\theta)_{\mathrm{eff}, \mathrm{bb}}$ and $R_{b}$ using numerical Mellin-Barnes integrals, JHEP 08 (2012) 050 [Erratum ibid. 1305 (2013) 074] [arXiv: 1205.0299] [INSPIRE].

[15] K. Agashe, R. Contino, L. Da Rold and A. Pomarol, A custodial symmetry for Z $b \bar{b}$, Phys. Lett. B 641 (2006) 62 [hep-ph/0605341] [INSPIRE].

[16] K. Agashe and R. Contino, Composite Higgs-Mediated FCNC, Phys. Rev. D 80 (2009) 075016 [arXiv:0906.1542] [INSPIRE]. 
[17] S.R. Coleman, J. Wess and B. Zumino, Structure of phenomenological Lagrangians. 1., Phys. Rev. 177 (1969) 2239 [InSPIRE].

[18] R. Contino, D. Marzocca, D. Pappadopulo and R. Rattazzi, On the effect of resonances in composite Higgs phenomenology, JHEP 10 (2011) 081 [arXiv:1109.1570] [INSPIRE].

[19] R. Barbieri, B. Bellazzini, V.S. Rychkov and A. Varagnolo, The Higgs boson from an extended symmetry, Phys. Rev. D 76 (2007) 115008 [arXiv:0706.0432] [INSPIRE].

[20] M. Farina, C. Grojean and E. Salvioni, (Dys)Zphilia or a custodial breaking Higgs at the LHC, JHEP 07 (2012) 012 [arXiv:1205.0011] [INSPIRE].

[21] A. Pomarol and J. Serra, Top quark compositeness: feasibility and implications, Phys. Rev. D 78 (2008) 074026 [arXiv:0806.3247] [InSPIRE].

[22] A. Azatov and J. Galloway, Light custodians and Higgs physics in composite models, Phys. Rev. D 85 (2012) 055013 [arXiv:1110.5646] [INSPIRE].

[23] G. Branco, P. Ferreira, L. Lavoura, M. Rebelo, M. Sher et al., Theory and phenomenology of two-Higgs-doublet models, Phys. Rept. 516 (2012) 1 [arXiv:1106. 0034] [INSPIRE].

[24] R. Slansky, Group theory for unified model building, Phys. Rept. 79 (1981) 1 [INSPIRE].

[25] F. Buccella, J. Derendinger, S. Ferrara and C.A. Savoy, Patterns of symmetry breaking in supersymmetric gauge theories, Phys. Lett. B 115 (1982) 375 [inSPIRE]. 